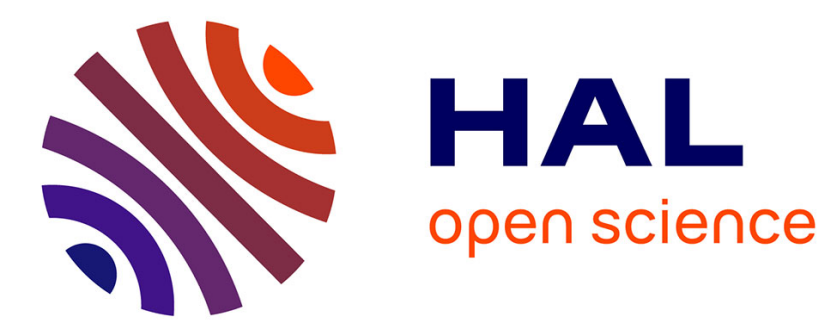

\title{
Computational Analysis of Foil Air Journal Bearings Using a Runtime-Efficient Segmented Foil Model
}

\author{
Tim Leister, Christoph Baum, Wolfgang Seemann
}

\section{To cite this version:}

Tim Leister, Christoph Baum, Wolfgang Seemann. Computational Analysis of Foil Air Journal Bearings Using a Runtime-Efficient Segmented Foil Model. 16th International Symposium on Transport Phenomena and Dynamics of Rotating Machinery, Apr 2016, Honolulu, United States. hal-01891326

\section{HAL Id: hal-01891326 \\ https://hal.science/hal-01891326}

Submitted on 9 Oct 2018

HAL is a multi-disciplinary open access archive for the deposit and dissemination of scientific research documents, whether they are published or not. The documents may come from teaching and research institutions in France or abroad, or from public or private research centers.
L'archive ouverte pluridisciplinaire HAL, est destinée au dépôt et à la diffusion de documents scientifiques de niveau recherche, publiés ou non, émanant des établissements d'enseignement et de recherche français ou étrangers, des laboratoires publics ou privés. 


\title{
Computational Analysis of Foil Air Journal Bearings Using a Runtime-Efficient Segmented Foil Model
}

\author{
Tim Leister ${ }^{1}$, Christoph Baum ${ }^{1}$, Wolfgang Seemann ${ }^{1}$ \\ \{tim.leister, christoph.baum,wolfgang.seemann\}@kit.edu
}

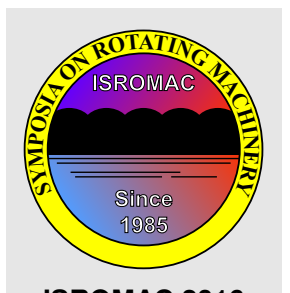

\begin{abstract}
This work is concerned with the development and the implementation of a foil air journal bearing model. For this purpose, the numerical procedure resolving the REYNOLDs equation for compressible fluids has to be coupled to a structural, compliant foil model. The presented beam-based approach is supposed to reproduce most of the experimentally known particularities in the mechanical behavior of the foil structure, while being at least as runtime-efficient as the commonly used simple elastic foundation model. The developed modeling approach will be validated by comparing simulation results to data found with a more complex reference model. In the analysis part, most notably, the top foil compliancy is shown to deteriorate the load-carrying capacity of air bearings. Moreover, the influence of the top foil compliancy
\end{abstract}

International

Symposium on Transport

Phenomena and Dynamics of Rotating Machinery Keywords Foil Air Journal Bearing, Foil Model, Load-Carrying Capacity, Rotor Dynamics

Hawaii, Honolulu

April 10-15, 2016

\section{${ }^{1}$ Institute of Engineering Mechanics, Karlsruhe Institute of Technology (KIT), Germany}

\section{INTRODUCTION}

Self-acting foil air journal bearings are an upcoming technology in high-speed rotating machinery and benefit from some major advantages compared to conventional rollingelement bearings. Most notably, the absence of solid-to-solid contact reduces both wear and power loss [1]. However, air bearing rotor systems may exhibit self-excited vibrations under certain conditions. As shown in [2], the insertion of a compliant foil structure into the lubrication gap seems to reduce this undesirable effect.

Currently, most foil models used for numerical investigations are based on one of two widespread approaches. The classical method neglects the segmentation of the foil structure and assumes it to act approximately as a homogeneous linear elastic foundation (see [1]), whereas numerous recent publications (see, e.g., [3]) rely on time-consuming FE models aiming at more realistic simulations.

The beam-based model presented in this paper is supposed to reproduce most of the experimentally known particularities (see [3]) in the static and transient behavior of the segmented foil structure, while being at least as runtime-efficient as the simple elastic foundation model.

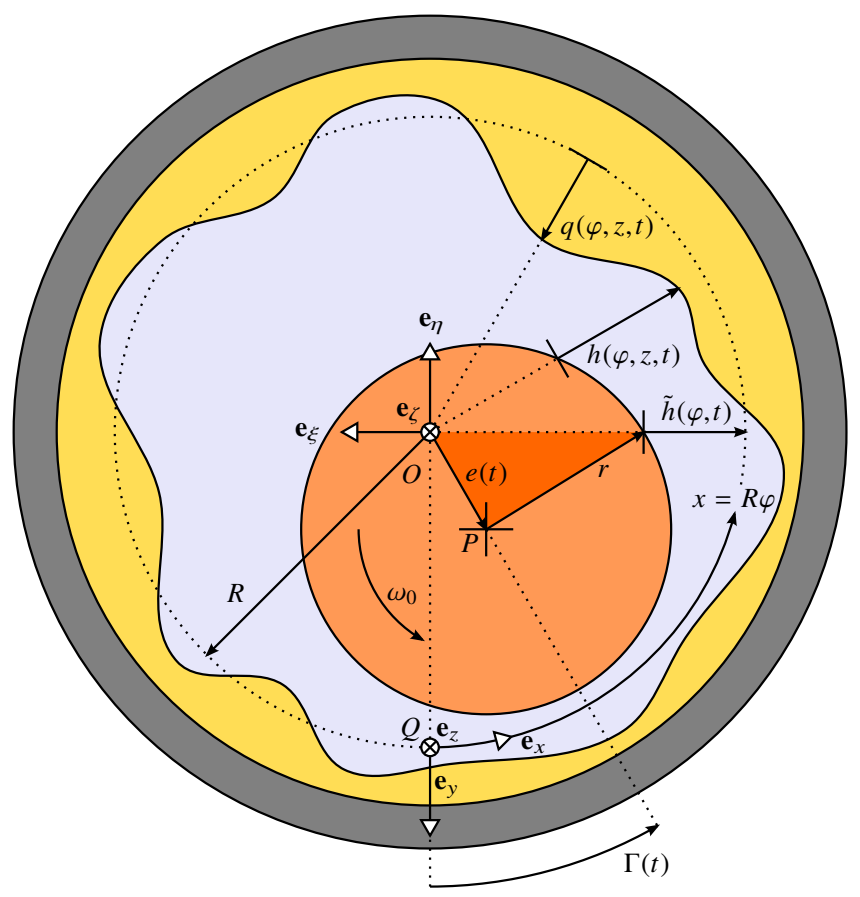

Figure 1. Sketch of the foil air journal bearing model. 


\section{THEORY}

\subsection{Bearing model}

Film thickness The nominal height of the lubrication gap between the bearing sleeve/foil (inner radius $R$, axial width $L$ ) and the rotating journal (outer radius $r$ ) can be characterized in terms of the radial clearance $C=R-r$. For visibility reasons, this gap is greatly magnified in the sketch depicted in figure 1. In order to determine the effective film thickness $h(\varphi, z, t)$ of the lubricant, we have to take into account the time-dependent journal position, i.e., $e(t)$ and $\Gamma(t)$, as well as the deflection of the compliant foil structure. At this point, the latter is dealt with as a generalized deformation field $q(\varphi, z, t)$, which will be discussed and concretized in section 1.3. By means of a simple trigonometric consideration based on the orange triangle marked in the sketch, a nondimensional form of the effective fluid film thickness is found to be approximately (linearized with respect to the radial journal displacement)

$$
H(\varphi, Z, \tau)=1-\varepsilon(\tau) \cos [\varphi-\gamma(\tau)]-Q(\varphi, Z, \tau)
$$

with the nondimensional quantities

$$
\begin{array}{ll} 
& \varphi=x / R, \quad Z=z / L, \quad \tau=t / T, \\
& \varepsilon(\tau)=e(\tau T) / C, \quad \gamma(\tau)=\Gamma(\tau T), \\
& H(\varphi, Z, \tau)=h(\varphi, Z L, \tau T) / C \\
\text { and } \quad & Q(\varphi, Z, \tau)=q(\varphi, Z L, \tau T) / C .
\end{array}
$$

Operating within the full fluid film lubrication regime, the load-carrying capacity of air bearings is limited with regard to the sustainment of a minimum film thickness throughout the gap. This condition can be expressed by

$$
H_{\min }(\tau)=\min _{(\varphi, Z) \in[0,2 \pi] \times\left[-1 / 2,+^{1 / 2}\right]} H(\varphi, Z, \tau)>H_{0},
$$

where $H_{0}$ is related to the surrounding surface roughnesses.

Reynolds equation The considered bearing uses ambient air with a constant dynamic viscosity $\mu_{0}$ as the lubricating fluid. As stated by the well-known lubrication theory, the pressure distribution $p(\varphi, z, t)$ within a thin gas film is governed by the REYNOLDS equation for compressible fluids [4]. Introducing a nondimensional pressure field $P=P(\varphi, Z, \tau)=$ $p(\varphi, Z L, \tau T) / p_{0}$ related to the constant ambient pressure $p_{0}$, we find the partial differential equation

$$
\frac{\partial}{\partial \varphi}\left(P H^{3} \frac{\partial P}{\partial \varphi}\right)+\kappa^{2} \frac{\partial}{\partial Z}\left(P H^{3} \frac{\partial P}{\partial Z}\right)=\Lambda \frac{\partial(P H)}{\partial \varphi}+2 \frac{\partial(P H)}{\partial \tau} .
$$

This equation involves the film thickness $H=H(\varphi, Z, \tau)$ coming from equation (1), as well as two nondimensional numbers

$$
\kappa=\frac{R}{L} \quad \text { and } \quad \Lambda=\frac{6 \mu_{0} \omega_{0}}{p_{0}}\left(\frac{R}{C}\right)^{2} .
$$

The bearing number $\Lambda$ represents the angular velocity $\omega_{0}$ of the journal, which is calculated from the rotational speed $n_{0}$ of the rotor by $\omega_{0}=2 \pi n_{0}$. The characteristic time scale of the nondimensional problem is defined as $T=6 \mu_{0} / p_{0}(R / C)^{2}$.
Boundary conditions and initial condition The resolution of equation (4) requires four boundary conditions and one initial condition. Assuming a continuous and differentiable pressure field, we use periodic boundary conditions in circumferential direction, giving

$$
\begin{aligned}
\left.P(\varphi, Z, \tau)\right|_{\varphi=0} & =\left.P(\varphi, Z, \tau)\right|_{\varphi=2 \pi}, \\
\left.\frac{\partial P(\varphi, Z, \tau)}{\partial \varphi}\right|_{\varphi=0} & =\left.\frac{\partial P(\varphi, Z, \tau)}{\partial \varphi}\right|_{\varphi=2 \pi} .
\end{aligned}
$$

In axial direction, the open edges of the lubrication gap are exposed to ambient pressure. Moreover, ambient pressure is supposed to be prevailing throughout the fluid at $t=\tau=0$. Thus, we have two additional boundary conditions

$$
\left.P(\varphi, Z, \tau)\right|_{Z=+1 / 2}=\left.P(\varphi, Z, \tau)\right|_{Z=-1 / 2}=1
$$

and the initial condition

$$
\left.P(\varphi, Z, \tau)\right|_{\tau=0}=\tilde{P}(\varphi, Z)=1
$$

\subsection{Rotor model}

Bearing force The rotor is supported by the time-dependent bearing force $\mathbf{F}(t)$, which can be calculated by integrating the pressure acting on the journal. Hence, the corresponding nondimensional force vector $\mathbf{f}(\tau)=\mathbf{F}(\tau T) /\left(p_{0} R L\right)$ is found to be

$$
\mathbf{f}(\tau)=\left(\begin{array}{l}
f_{\xi}(\tau) \\
f_{\eta}(\tau)
\end{array}\right)_{\left\{\mathbf{e}_{\xi}, \mathbf{e}_{\eta}\right\}}=\int_{-1 / 2}^{+1 / 2} \int_{0}^{2 \pi} P(\varphi, Z, \tau)\left(\begin{array}{c}
\sin \varphi \\
\cos \varphi
\end{array}\right)_{\left\{\mathbf{e}_{\xi}, \mathbf{e}_{\eta}\right\}} \mathrm{d} \varphi \mathrm{d} Z
$$

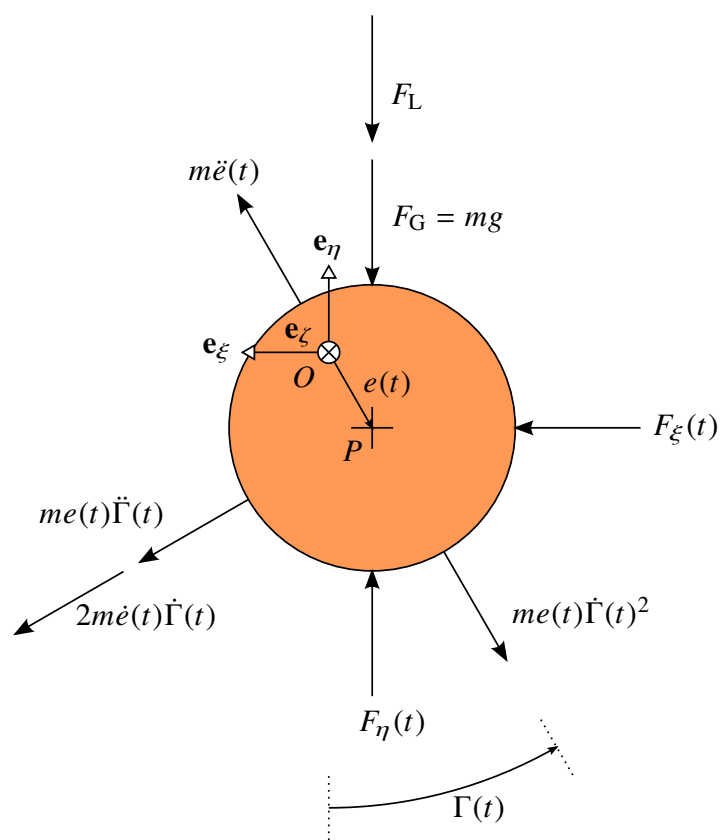

Figure 2. Free body diagram of the journal. 


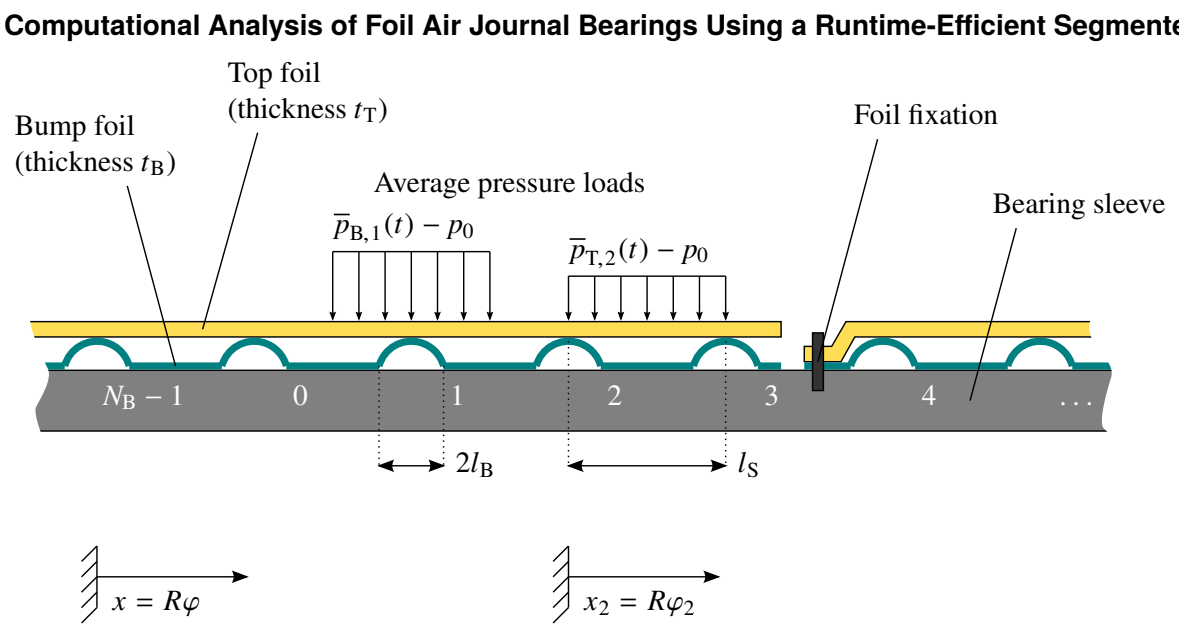

Figure 3. Configuration of the compliant foil structure.

Equations of motion In this study, a rigid rotor of mass $2 m$ without unbalance is considered. Symmetrically mounted on two bearings, the journals on each side are exposed to the gravitational force $m g$, the vertical load $F_{\mathrm{L}}$ and the bearing force components $F_{\xi}(t)$ and $F_{\eta}(t)$. In figure 2, a free body diagram of the journal is depicted. When equilibrating all external forces and inertia forces, we find the nondimensional equations of motion

$$
\begin{aligned}
& \varepsilon^{\prime \prime}(\tau)-\varepsilon(\tau) \gamma^{\prime}(\tau)^{2}= \\
& G \cos \gamma(\tau)+\frac{1}{M}\left[-f_{\xi}(\tau) \sin \gamma(\tau)-\left(f_{\eta}(\tau)-f_{\mathrm{L}}\right) \cos \gamma(\tau)\right], \\
& \varepsilon(\tau) \gamma^{\prime \prime}(\tau)+2 \varepsilon^{\prime}(\tau) \gamma^{\prime}(\tau)= \\
& -G \sin \gamma(\tau)+\frac{1}{M}\left[-f_{\xi}(\tau) \cos \gamma(\tau)+\left(f_{\eta}(\tau)-f_{\mathrm{L}}\right) \sin \gamma(\tau)\right]
\end{aligned}
$$

with two nondimensional parameters

$$
M=\frac{p_{0}}{36 \mu_{0}^{2} L}\left(\frac{C}{R}\right)^{5} m \text { and } G=\frac{36 \mu_{0}^{2}}{p_{0}^{2} R}\left(\frac{R}{C}\right)^{5} g .
$$

For the calculation of $\varepsilon(\tau)$ and $\gamma(\tau)$, four initial conditions are required and will be defined as

$$
\begin{array}{ll}
\left.\varepsilon(\tau)\right|_{\tau=0}=\varepsilon_{0}, & \left.\varepsilon^{\prime}(\tau)\right|_{\tau=0}=\varepsilon_{0}^{\bullet}, \\
\left.\gamma(\tau)\right|_{\tau=0}=\gamma_{0}, & \left.\gamma^{\prime}(\tau)\right|_{\tau=0}=\gamma_{0}^{\bullet}
\end{array}
$$

with given $\varepsilon_{0}, \varepsilon_{0}^{\bullet}, \gamma_{0}, \gamma_{0}^{\bullet}$.

\subsection{Foil model}

Segmented structure Figure 3 shows the configuration of the compliant foil structure typically used in first generation foil air journal bearings. It is composed of a thin, corrugated bump foil (thickness $t_{\mathrm{B}}$, Young's modulus $E_{\mathrm{B}}$, Poisson's ratio $v_{\mathrm{B}}$ ) and a thin, smooth top foil (thickness $t_{\mathrm{T}}$, YounG's modulus $E_{\mathrm{T}}$, Poisson's ratio $v_{\mathrm{T}}$ ). The bump foil consists of $N_{\mathrm{B}}$ equally spaced bumps, giving the nondimensional bump distance or top foil segment width

$$
L_{\mathrm{S}}=\frac{l_{\mathrm{S}}}{R}=\frac{2 \pi}{N_{\mathrm{B}}} .
$$

Within the structure, relative motion or detachment between the bump foil and the top foil are possible. They are only constrained at one end, where both foil components are welded to the bearing sleeve. However, this study is focused on the foil deflection and ignores these effects for the sake of simplicity.

Fluid-structure interaction In foil air journal bearings, the pressurized fluid inside the lubrication gap interacts with both the journal and the foil structure. When neglecting inertia effects for the latter because of the thin foil geometry, the deformation field $q(\varphi, z, t)$ at any given time can be deduced directly from the current pressure distribution $p(\varphi, z, t)$. On the other hand, the foil deflection affects the pressure field via the REYNOLDS equation. It has been shown that the behavior of the compliant foil structure can be represented in good approximation by a plane model with respect to the axial direction [3]. In this context, we are furthermore interested in the calculation of a nondimensional plane deformation field $Q(\varphi, Z, \tau)=\bar{Q}(\varphi, \tau)$ as a function of $P(\varphi, Z, \tau)$, which can be inserted into equation (1).

Average pressure loads Working henceforth with plane foil models, we define an average pressure function with respect to the axial direction, giving

$$
\bar{P}(\varphi, \tau)=\int_{-1 / 2}^{+1 / 2} P(\varphi, Z, \tau) \mathrm{d} Z .
$$

Taking advantage of the segmented foil structure, we introduce discrete pressure loads with respect to the circumferential direction. As marked in the sketch depicted in figure 3, we define the average pressure acting on the $n$-th bump

$$
\bar{P}_{\mathrm{B}, n}(\tau)=\frac{1}{L_{\mathrm{S}}} \int_{(n-1 / 2)}^{(n+1 / 2) L_{\mathrm{S}}} \int_{-1 / 2}^{L_{\mathrm{S}}} P(\varphi, Z, \tau) \mathrm{d} Z \mathrm{~d} \varphi
$$

and the average pressure acting on the $n$-th top foil segment

$$
\bar{P}_{\mathrm{T}, n}(\tau)=\frac{1}{L_{\mathrm{S}}} \int_{n L_{\mathrm{S}}}^{(n+1)} \int_{-1 / 2}^{L_{\mathrm{S}}} P(\varphi, Z, \tau) \mathrm{d} Z \mathrm{~d} \varphi .
$$




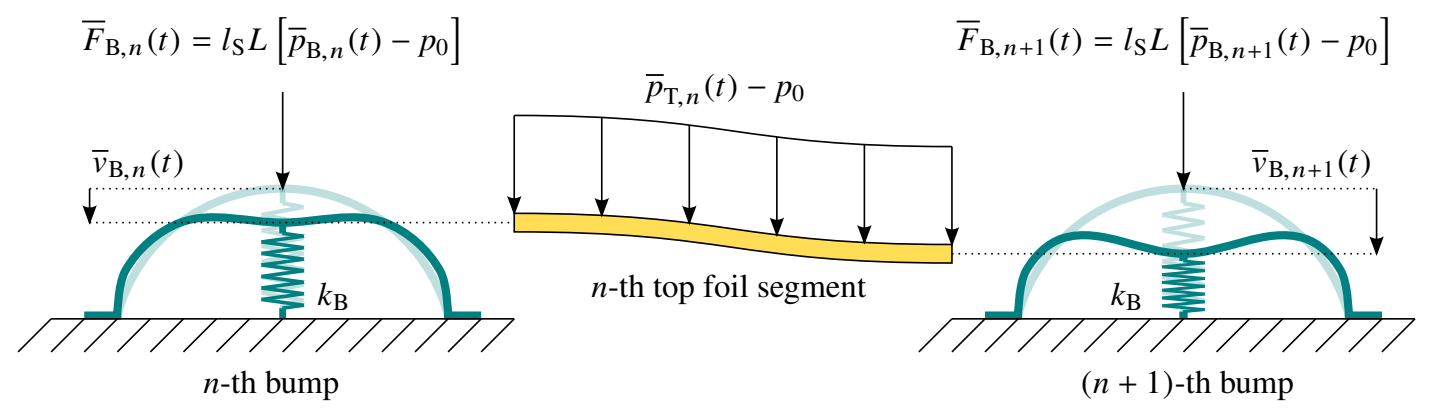

Figure 4. Sketch of the segmented foil model.

Beam theory Both the bump foil and the top foil will be rep esen ed beam-b sed models. According to the EuLERBERnOulu theory, we establish the differential equation

$$
\frac{\mathrm{d}^{4} \bar{V}(\varphi, \tau)}{\mathrm{d} \varphi^{4}}=-\frac{\bar{P}(\varphi, \tau)-1}{\delta}
$$

for the determination of the nondimensional deflection curve $\bar{V}(\varphi, \tau)=\bar{v}(\varphi, \tau T) / C$. The stiffness is expressed by the nondien nsional flexural rigidity $\delta=E t^{3} C /\left[12\left(1-v^{2}\right) p_{0} R^{4}\right]$.

Bump stiffness The bumps will be modeled as linear elastic springs. It has been shown that their curved geometry can be appo ximated by means of a simple beam model [5], which reveals the nondimensional equivalent spring stiffness

$$
K_{\mathrm{B}}=\frac{E_{\mathrm{B}} C}{2\left(1-v_{\mathrm{B}}^{2}\right) p_{0} R}\left(\frac{t_{\mathrm{B}}}{l_{\mathrm{B}}}\right)^{3} .
$$

From this, we can calculate the deformation

$$
\bar{V}_{\mathrm{B}, n}(\tau)=\frac{\bar{f}_{\mathrm{B}, n}(\tau)}{K_{\mathrm{B}}}
$$

e sulting $6 \mathrm{~m}$ a nondimensional vertical force $\bar{f}_{\mathrm{B}, n}(\tau)=$ $\bar{F}_{\mathrm{B}, n}(\tau T) /\left(p_{0} R L\right)$ acting on the apex of the $n$-th bump.

Simple elastic foundation model A commonly used modeling approach in the available literature considers the foil structure as a simple, homogeneous elastic foundation [1]. In this work, we will refer to a slightly modified version of this model, in which the def $\mathrm{m}$ ation field does not vary in axial direction. The actual displacement is assumed to be proportional to the average pressure, calculated by equation (14), giving

$$
\bar{Q}_{\mathrm{W}}(\varphi, \tau)=-\frac{\bar{P}(\varphi, \tau)-1}{K_{\mathrm{W}}} .
$$

The nondien nsional foundation stiffness

$$
K_{\mathrm{W}}=\frac{E_{\mathrm{B}} C}{2\left(1-v_{\mathrm{B}}^{2}\right) p_{0} R L_{\mathrm{S}}}\left(\frac{t_{\mathrm{B}}}{l_{\mathrm{B}}}\right)^{3}=\frac{K_{\mathrm{B}}}{L_{\mathrm{S}}}
$$

is deduced from the nondimensional bump stiffness $K_{\mathrm{B}}$ stated by equation (18) and does not take into account any of the properties of the top foil. The displacements calculated with the simple elastic foundation model are completely decoupled in circumferential direction. In a certain way, the use of a plane model imitates a coupled deformation in axial direction and prevents non-physical contact between the journal and th tp fo 1 at th $p$ n b arig ed $s, d$ sp te th p evailig ambient pressure.

Runtime-efficient segmented model The fundamental idea behind the presented runtime-efficient model is to decouple the bump foil from the top foil, the latter being furthermore divided into separated segments. In a first step, each bump is then considered independently and the corresponding spring displacement $\bar{V}_{\mathrm{B}, n}(\tau)$ is estimated by the use of equation (19) with the acting force (deduced from equation (15))

$$
\bar{f}_{\mathrm{B}, n}(\tau)=L_{\mathrm{S}}\left[\bar{P}_{\mathrm{B}, n}(\tau)-1\right] \text {. }
$$

In a second step, illustrated by figure 4 , each top foil segment is modeled as a beam charged by $\bar{P}_{\mathrm{T}, n}(\tau)$ (see equation (16)). Local coordinates for the description of the $n$-th top foil segment are introduced by $\varphi_{n}=\varphi-2 \pi n / N_{\mathrm{B}}$. With the already known bump deformations, we set displacement boundary conditions for the beam's deflection curve $\bar{V}_{\mathrm{S}, n}\left(\varphi_{n}, \tau\right)$, giving

$$
\begin{aligned}
\left.\bar{V}_{\mathrm{S}, n}\left(\varphi_{n}, \tau\right)\right|_{\varphi_{n}=0} & =\bar{V}_{\mathrm{B}, n}(\tau), \\
\left.\bar{V}_{\mathrm{S}, n}\left(\varphi_{n}, \tau\right)\right|_{\varphi_{n}=L_{\mathrm{S}}} & =\bar{V}_{\mathrm{B}, n+1}(\tau) .
\end{aligned}
$$

Moreover, we suppose that the shape of the top foil adapts to the supporting horizontal bump apices and set

$$
\left.\frac{\mathrm{d} \bar{V}_{\mathrm{S}, n}\left(\varphi_{n}, \tau\right)}{\mathrm{d} \varphi_{n}}\right|_{\varphi_{n}=0}=\left.\frac{\mathrm{d} \bar{V}_{\mathrm{S}, n}\left(\varphi_{n}, \tau\right)}{\mathrm{d} \varphi_{n}}\right|_{\varphi_{n}=L_{\mathrm{S}}}=0
$$

By integrating equation (17) for each segment with these boundary conditions, we obtain a piecewise deflection curve

$$
\begin{aligned}
\bar{V}_{\mathrm{S}, n}\left(\varphi_{n}, \tau\right)=\frac{\bar{P}_{\mathrm{B}, n}(\tau)-1}{K_{\mathrm{W}}} & -\frac{3}{K_{\mathrm{W}}}\left[\bar{P}_{\mathrm{B}, n}(\tau)-\bar{P}_{\mathrm{B}, n+1}(\tau)\right]\left(\frac{\varphi_{n}}{L_{\mathrm{S}}}\right)^{2} \\
& +\frac{2}{K_{\mathrm{W}}}\left[\bar{P}_{\mathrm{B}, n}(\tau)-\bar{P}_{\mathrm{B}, n+1}(\tau)\right]\left(\frac{\varphi_{n}}{L_{\mathrm{S}}}\right)^{3} \\
& +\frac{\bar{P}_{\mathrm{T}, n}(\tau)-1}{24 \delta_{\mathrm{T}}} \varphi_{n}^{2}\left(L_{\mathrm{S}}-\varphi_{n}\right)^{2} .
\end{aligned}
$$




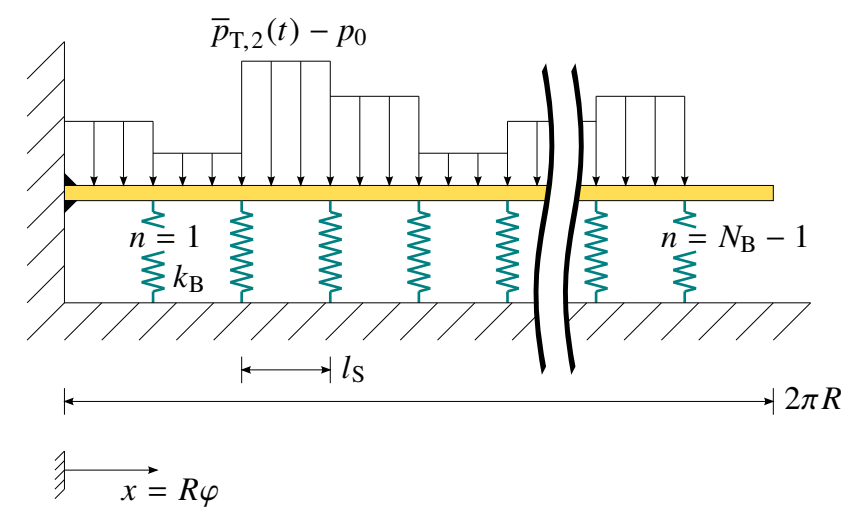

Figure 5. Sketch of the reference foil model.

The deformation field is eventually found to be a combination of the piecewise deflection curves from equation (25) and can be stated as a function of the global coordinate $\varphi$ by

$$
\bar{Q}_{\mathrm{S}}(\varphi, \tau)=-\bar{V}_{\mathrm{S},\left\lfloor N_{\mathrm{B}} \frac{\varphi}{2 \pi}\right\rfloor}\left(\varphi-2 \pi \frac{\left\lfloor N_{\mathrm{B}} \frac{\varphi}{2 \pi}\right\rfloor}{N_{\mathrm{B}}}, \tau\right)
$$

with the floor function $\left\lfloor N_{\mathrm{B}} \frac{\varphi}{2 \pi}\right\rfloor$ for finding the correct segment.

Reference model In order to validate the segmented foil model, a reference model (similar to models found in the available literature [3]) is implemented. A self-explanatory sketch is depicted in figure 5, showing the top foil modeled as a cantilever beam, which is supported by multiple springs. In this model, the coupled deformation of the beam and the springs is calculated without introducing any kind of cutoff distance. Thus, the pressure at any given point even affects the deformation field on the opposite side of the bearing, resulting in increased computational times.

\section{ANALYSIS}

Discretized state vector The domain of the lubrication gap $(\varphi, Z) \in[0,2 \pi] \times\left[-1 / 2,+^{1} / 2\right]$ is discretized using a uniform computational grid with $N_{\varphi} \times N_{Z}$ grid points. As for the numerical analysis, the discrete pressure values as well as the

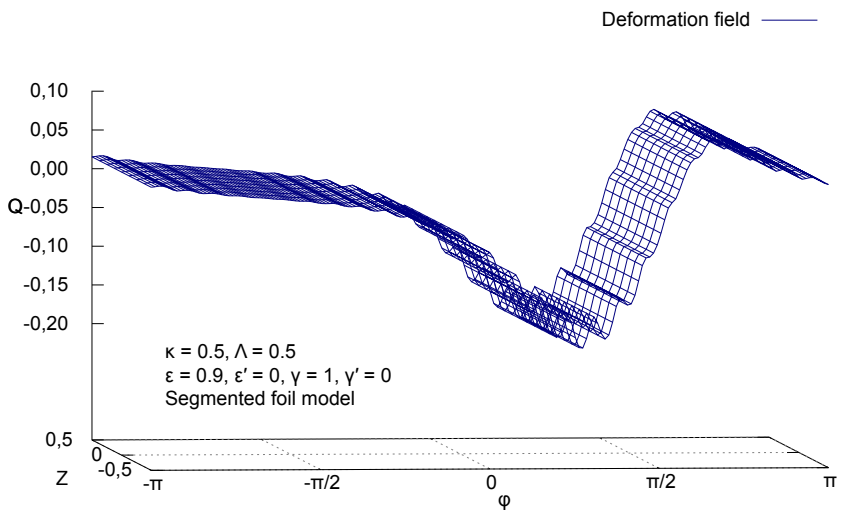

Figure 6. Foil deformation (using segmented foil model). journal state variables are collected in a nondimensional and discretized state vector

$\mathbf{S}(\tau)=\left(\begin{array}{lllllll}\varepsilon(\tau) & \varepsilon^{\prime}(\tau) & \gamma(\tau) & \gamma^{\prime}(\tau) & P_{0,0}(\tau) & \ldots & P_{N_{\varphi}-1, N_{Z}-1}(\tau)\end{array}\right)^{\top}$.

Due to the negligence of the foil structure's inertia effects, the discrete deformation values are not directly included in this state vector. Instead of that, they can be deduced from the current pressure distribution at any given time, using either equation (20), equation (26) or the reference model. The latter is analytically evaluated with MAPLE, due to its rather complex deflection curve.

Time integration scheme The differential equation for the state vector $\mathrm{d} \mathbf{S}(\tau) / \mathrm{d} \tau=\mathbf{k}(\mathbf{S}(\tau))$ is resolved using an explicit EULER method as the time integration scheme. The function $\mathbf{k}$ is deduced from equation (4) and equation (10). Most notably, we establish a spatially discretized form of the REYNOLDS equation, giving

$$
\begin{aligned}
\frac{\mathrm{d} P_{i, j}(\tau)}{\mathrm{d} \tau}=\frac{1}{2 H_{i, j}(\tau)} & {\left[\frac{\delta}{\delta \varphi}\left(P_{i, j}(\tau) H_{i, j}(\tau)^{3} \frac{\delta P_{i, j}(\tau)}{\delta \varphi}\right)\right.} \\
& +\kappa^{2} \frac{\delta}{\delta Z}\left(P_{i, j}(\tau) H_{i, j}(\tau)^{3} \frac{\delta P_{i, j}(\tau)}{\delta Z}\right) \\
& -\Lambda \frac{\delta\left(P_{i, j}(\tau) H_{i, j}(\tau)\right)}{\delta \varphi} \\
& +2 P_{i, j}(\tau)\left[\varepsilon^{\prime}(\tau) \cos (i \Delta \varphi-\gamma(\tau))\right. \\
& \left.\left.+\varepsilon(\tau) \gamma^{\prime}(\tau) \sin (i \Delta \varphi-\gamma(\tau))\right]\right]
\end{aligned}
$$

with $\frac{\delta(\ldots)_{i, j}}{\delta \varphi}=\frac{(\ldots)_{i+1, j}-(\ldots)_{i-1, j}}{2 \Delta \varphi}, \frac{\delta(\ldots)_{i, j}}{\delta Z}=\frac{(\ldots)_{i, j+1}-(\ldots)_{i, j-1}}{2 \Delta Z}$.

The described concepts are implemented as a $\mathrm{C}++$ program.

\section{RESULTS}

\subsection{Configuration}

Model parameters In this section, some numerical simulation results will be shown and discussed. Table 1 lists the data for a first generation test bearing, which is commonly referred to in the available literature (see, e.g., [3]). These parameters are converted to nondimensional values, according to the formulae presented in section 1 , and will be used as nominal values for the following investigations.

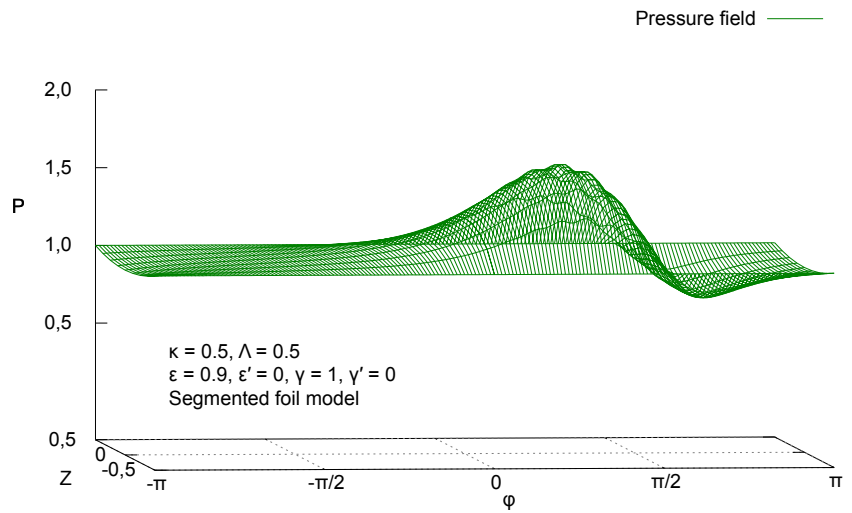

Figure 7. Pressure distribution (using segmented foil model). 


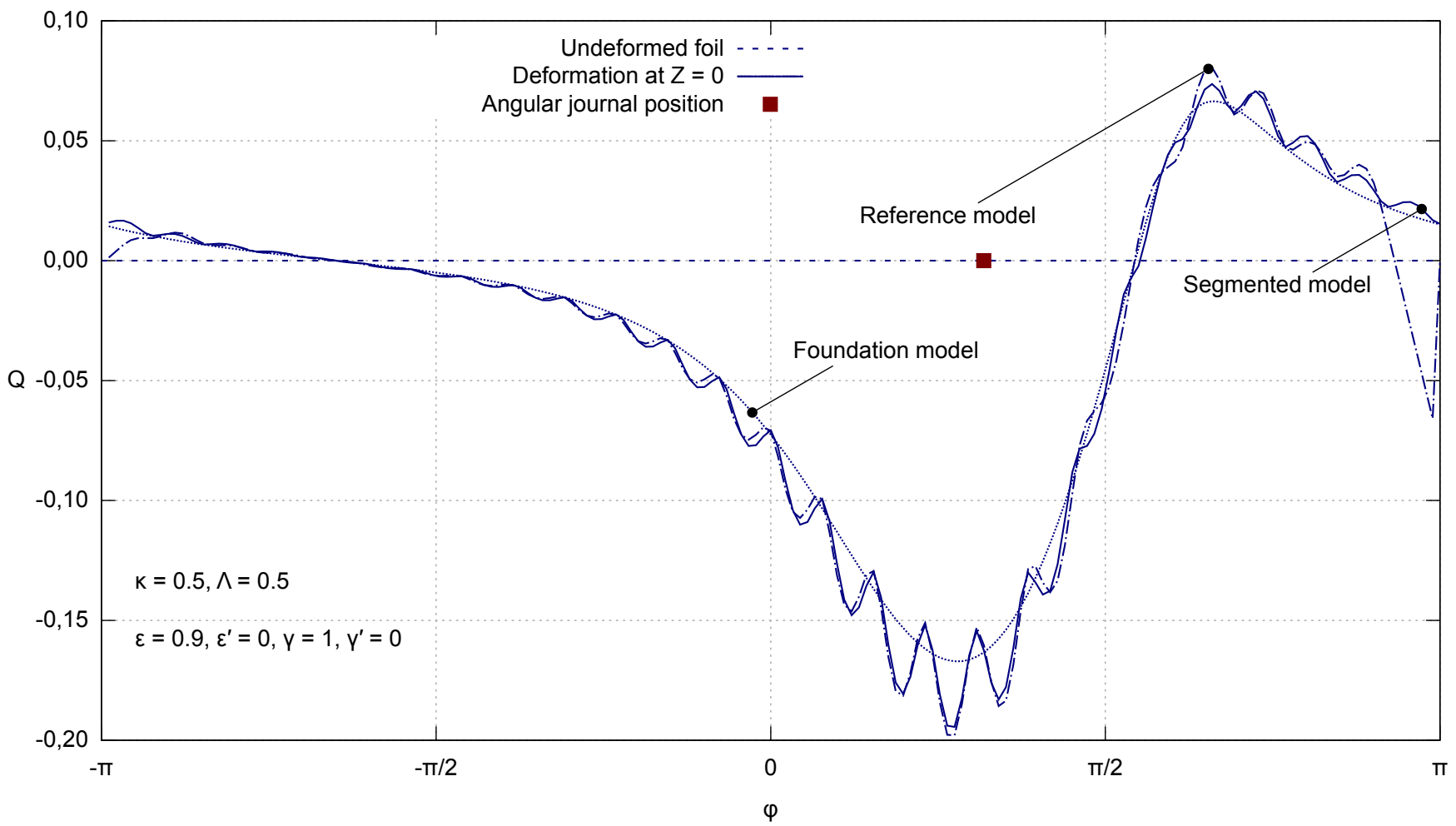

Figure 8. Foil deformation along the bearing centerline for different foil models.

Grid dependency study As a preliminary investigation, we have carried out a grid dependency study for the numerical solution of the REYNOLDs equation. For a rigid bearing, the pressure distribution found with $41 \times 9$ grid points does not change significantly after a further refinement. However, the segmented foil model is supposed to induce important film thickness variations within the scale of one segment width (see figure 6). In order to capture the reaction of the pressure distribution to this effect, we use $183 \times 9$ grid points in the considered case with $N_{\mathrm{B}}=26$ bumps. As expected, the pressure field depicted in figure 7 reveals local pressure drops resulting from the top foil sagging between the bumps. Due to fewer calculations of the average pressure, the segmented foil model needs less computational time than the plane, simple elastic foundation model on the same grid (40 s vs. $50 \mathrm{~s}$ using an Intel ${ }^{\circledR}$ Core $^{\mathrm{TM}} \mathrm{i} 5 \mathrm{CPU}$ for the calculations in section 3.2).

\subsection{Comparison of the foil models}

Approach In this investigation, the journal remains at the constant position $\varepsilon(\tau)=0.9, \gamma(\tau)=1$ and the equations of motion are not taken into consideration (commonly referred to as a quasi-stationary simulation). The time integration of the REYNOLDs equation is then carried out until a converged equilibrium state is reached. Subsequently, the predictions for the deformation field, the film thickness and the pressure distribution can be compared for the different foil models.

Deformation The plot in figure 8 visualizes the deformation field as predicted by the different foil models. For obvious reasons, the maximum deformation appears in the region of maximum pressure for the marked journal position (dark red square). Due to the assumption of a uniform stiffness, the simple elastic foundation model (dotted line) generates a smooth curve, which represents only the compliancy of the bump foil. Being based on the same bump stiffness definition, both the runtime-efficient segmented model (solid line) and the reference model (chain dotted line) predict deformations in the same order of magnitude. Apparently, the use of uncoupled deformations in circumferential direction

\begin{tabular}{llr}
\hline Parameter & Symbol & Value \\
\hline Axial bearing width & $L$ & $38.10 \mathrm{~mm}$ \\
Bearing radius & $R$ & $19.05 \mathrm{~mm}$ \\
Lubrication gap clearance & $C$ & $50 \mu \mathrm{m}$ \\
\hline Ambient pressure & $p_{0}$ & $1013.25 \mathrm{hPa}$ \\
Dynamic viscosity & $\mu_{0}$ & $1.85 \times 10^{-5} \mathrm{Ns} / \mathrm{m}^{2}$ \\
\hline Rotational speed & $n_{0}$ & $500 \mathrm{~s}^{-1}$ \\
Rotor mass & $2 m$ & $2 \times 185 \mathrm{~g}$ \\
Gravity & $g$ & $9.81 \mathrm{~m} / \mathrm{s}^{2}$ \\
External load & $2 F_{\mathrm{L}}$ & $2 \times 30 \mathrm{~N}$ \\
\hline Number of bumps & $N_{\mathrm{B}}$ & 26 \\
Bump width & $2 l_{\mathrm{B}}$ & $2 \times 1.778 \mathrm{~mm}$ \\
Foil thickness & $t_{\mathrm{B}}, t_{\mathrm{T}}$ & $101.6 \mu \mathrm{m}$ \\
YounG's modulus & $E_{\mathrm{B}}, E_{\mathrm{T}}$ & $214 \mathrm{GPa}$ \\
Poisson's number & $\nu_{\mathrm{B}}, \nu_{\mathrm{T}}$ & 0.29 \\
\hline
\end{tabular}

Table 1. Parameters used for the numerical simulations. 


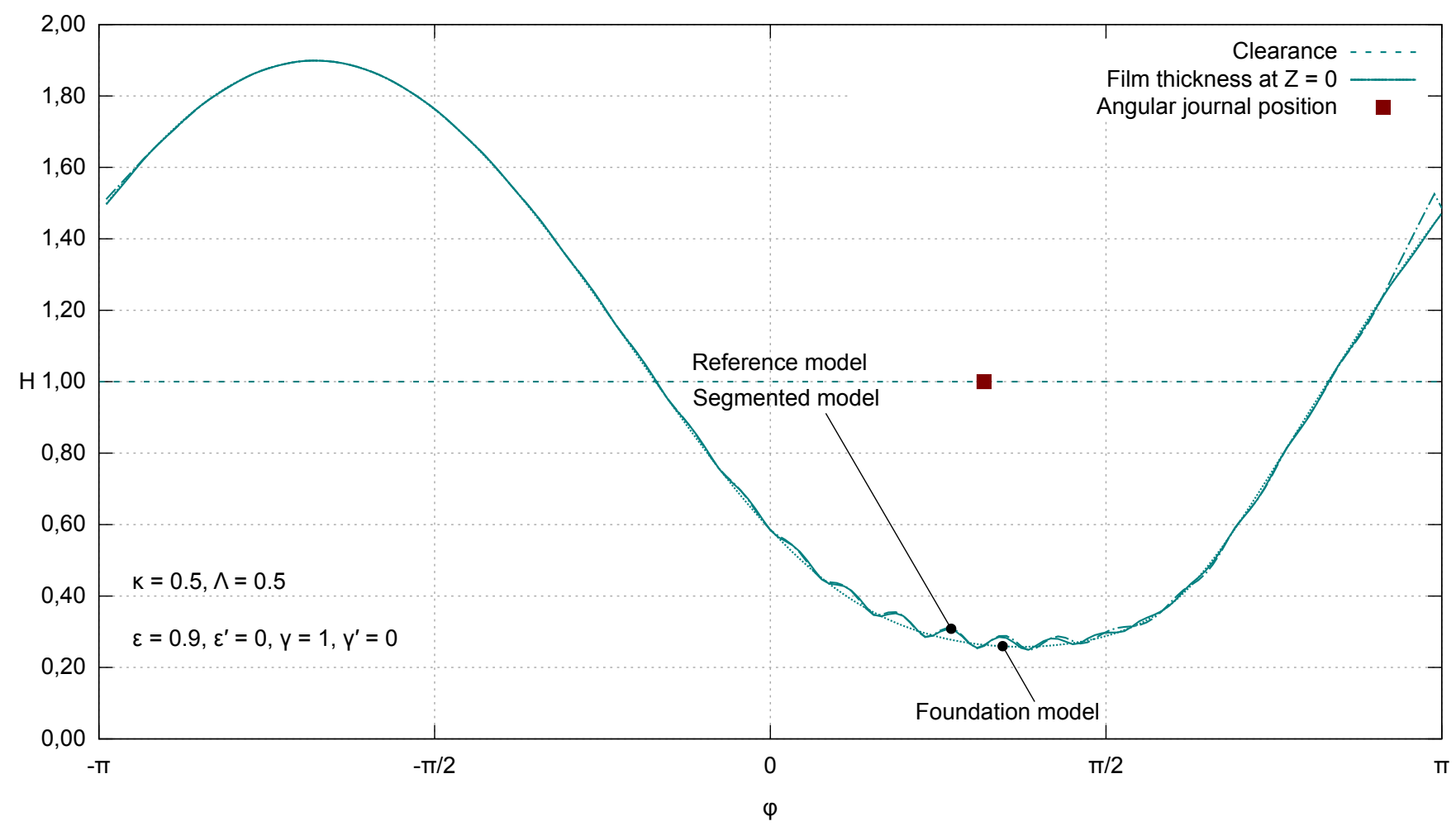

Figure 9. Film thickness along the bearing centerline for different foil models.
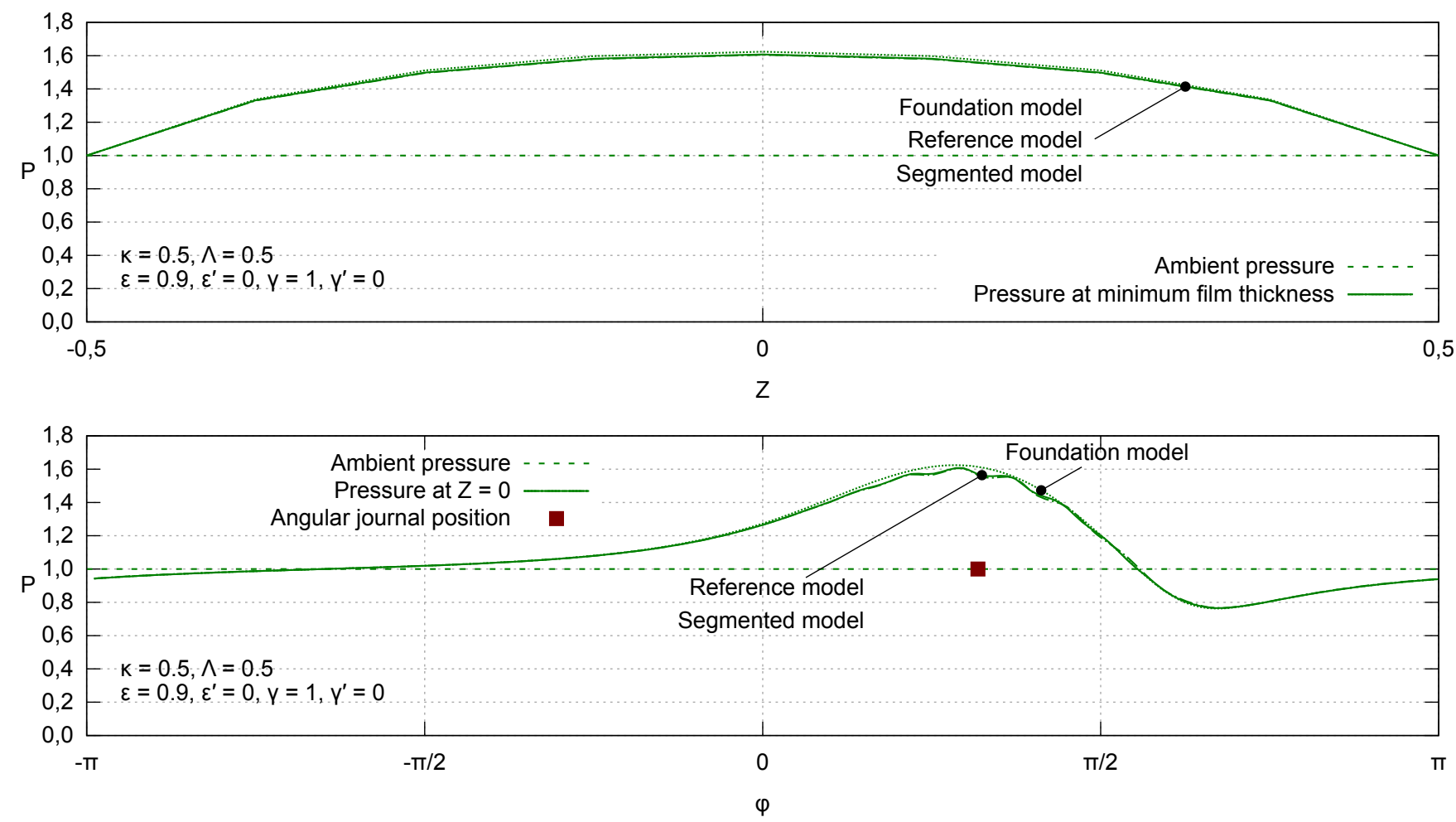

Figure 10. Pressure distribution in axial direction and along the bearing centerline for different foil models. 


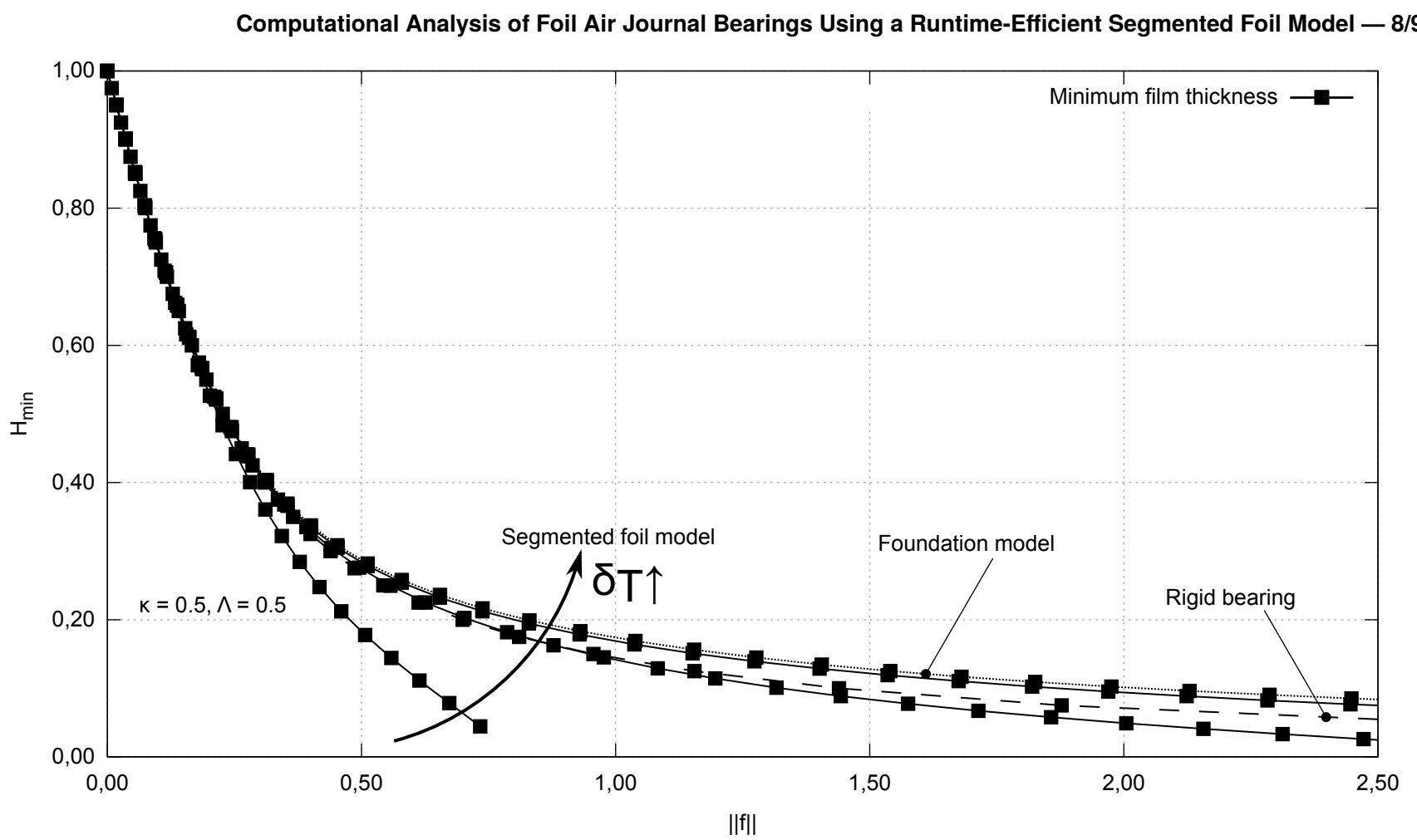

Figure 11. Effect of the foil structure on the load-carrying capacity.

does not induce crucial inaccuracies within the simple elastic foundation model. Moreover, the coincidence between the deformation field predicted by the segmented model and the one predicted by the reference model proves that the simplifying assumptions behind the former are reasonable. Besides the ripple-like effect resulting from the top foil sagging between the bumps, both models estimate slightly smaller bump displacements as a result of the local pressure drops.

Film thickness The plot in figure 9 shows the resulting film thickness for all three foil models. As stated by equation (1), the effective film thickness of the lubricating fluid depends on both the journal position and the deformation of the foil structure. For the simple elastic foundation model (dotted line), the curve's appearance is only slightly different from the cosine function known from rigid bearings. The segmented model (solid line) and the reference model (chain dotted line) show the additional effect of the top foil deformation. Being only visible in the thinnest zone of the lubrication gap, the global importance of this ripple-like effect seems to be small compared to the journal position's influence.

Pressure The plot in figure 10 depicts the pressure field for the different models. For the simple elastic foundation model (dotted line), the shape of the pressure distribution is similar to the results known from rigid bearings. The curves corresponding to the segmented model (solid line) and the reference model (chain dotted line) are once again very similar. Both of them show the already mentioned zones of local pressure drops, resulting from the top foil sagging between the bumps, which opens a pathway for the lubricating fluid to escape out of the gap, thus decreasing the pressure.

\subsection{Load-carrying capacity}

Approach As stated by equation (3), the load-carrying capacity of foil air journal bearings is limited by the surface roughnesses, meaning that the minimum film thickness must not fall below a certain limit $H_{0}$ for a given load. Thus, load-carrying capacity curves are supposed to show the minimum film thickness in the lubrication gap as a function of the bearing force. In this investigation, such curves are established by carrying out multiple simulations (each one of them is represented by a black square on the corresponding curve). During these simulations, the journal is moved step-by-step along the radial direction, calculating each time both the minimum film thickness and the resulting bearing force.

Effect of the foil structure The plot in figure 11 shows that the simple elastic foundation model (dotted line) predicts the best load-carrying capacity, being even higher than the one found for a rigid bearing model (dashed line), in particular for high loads. On the other hand, the load-carrying capacity curves found with the segmented foil model (solid lines) cover a wide performance range. While the one found with nominal parameter values is situated even below the curve of a rigid bearing, another simulation with doubled top foil thickness exhibits a load-carrying capacity almost as good as the simple elastic foundation model does. We deduce from this observation that the escaping air plays a crucial role and that the top foil compliancy has a detrimental effect on the loadcarrying capacity of the bearing. As to simulations carried out with the simple elastic foundation model, the load-carrying capacity seems to be systematically overestimated. 


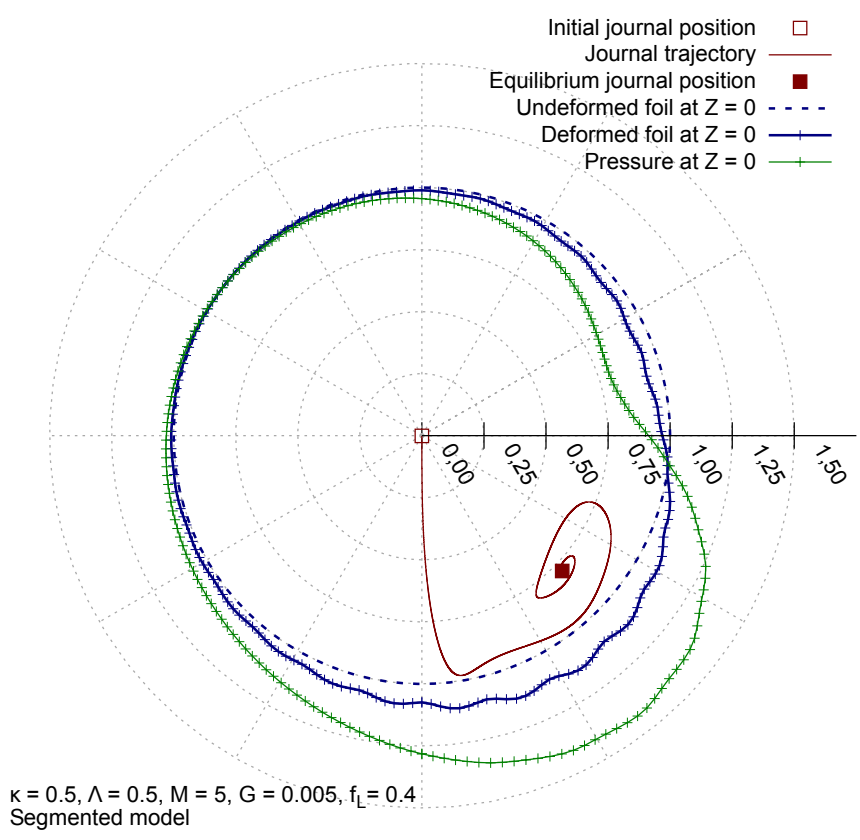

(a) Nominal top foil thickness.

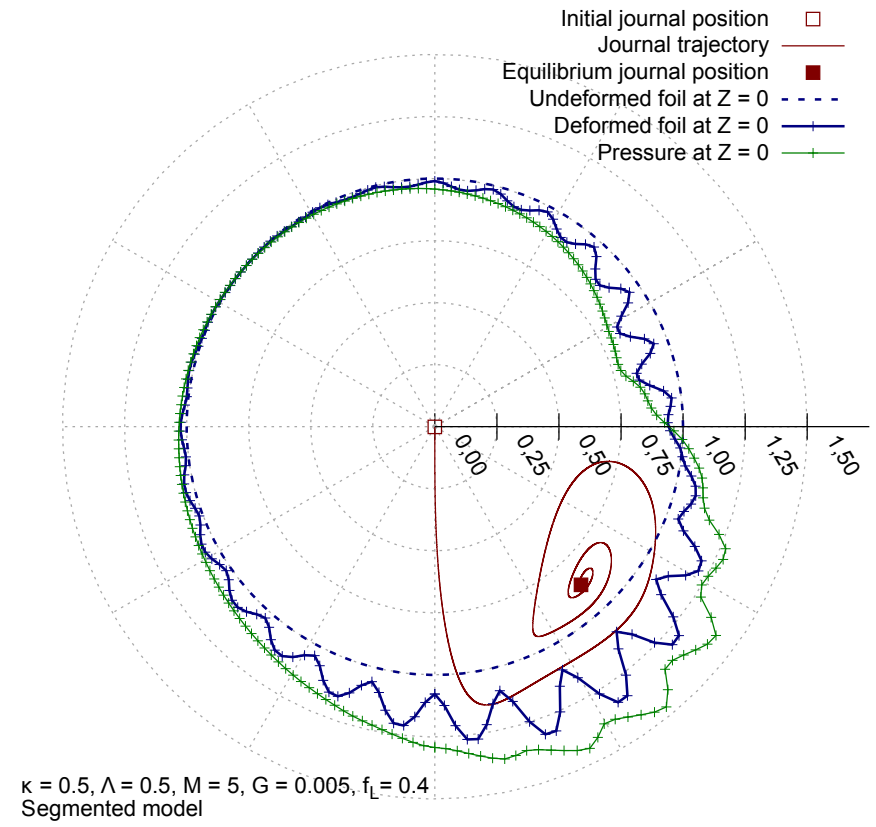

(b) Reduced top foil thickness.

Figure 12. Influence of the top foil stiffness on the journal's trajectory.

\subsection{Journal trajectory}

Effect of the foil structure The plot in figure 12 a shows the journal's trajectory after dropping the rotor from the center position using nominal parameter values. Again, both the deformation and the pressure field exhibit a ripple-like shape (shown for the equilibrium position). In a second simulation, we decrease the top foil stiffness and obtain the modified trajectory which is depicted in figure $12 \mathrm{~b}$. Resulting from the deteriorated bearing force caused by larger top foil deflections, the second trajectory covers a broader area and takes more time to reach the equilibrium position, which is also farther away from the bearing center. Thus, we conclude that the top foil compliancy has to be taken into consideration in order to carry out realistic transient rotor dynamics simulations.

\section{CONCLUSION}

The developed and implemented segmented foil model permits us to perform static and transient simulations of foil air journal bearings. In contrast to the commonly used simple elastic foundation, this model also takes into account the top foil sagging between the bumps and the resulting ripple-like film thickness variations. The observed local pressure drops can be explained by the air escaping out of the bearing as the top foil is deflected. Thanks to the piecewise calculation of beam deflection curves, the established model turns out to be more runtime-efficient than the simple elastic foundation model. Most notably, we have shown that the compliancy of the top foil has a detrimental effect on the bearing's loadcarrying capacity. Thus, simulations with the simple elastic foundation model tend to overestimate the bearing force. Moreover, we have seen that the rotor's transient behavior is crucially influenced by this effect.
Further investigations will consider friction and damping effects coming from relative motion between the top foil, the bump foil and the bearing sleeve. Besides, the foil behavior at the open bearing edges may be studied in order to validate the assumption of a plane model.

\section{REFERENCES}

[1] H. Heshmat et al. (1983).

Analysis of Gas-Lubricated Foil Journal Bearings.

Journal of Lubrication Technology, Vol. 105.

[2] C. Baum et al. (2015).

On the Stability of Balanced Rigid Rotors in Air Foil Bearings. SIRM 2015, Magdeburg, Germany.

[3] L. SAN ANDRÉs et al. (2008).

Analysis of gas foil bearings integrating FE top foil models.

Tribology International, Vol. 42.

[4] A. SZERI (2011).

Fluid film lubrication.

$2^{\text {nd }}$ edition.

Cambridge University Press.

[5] J. A. Walowit et al. (1975).

Modern developments in lubrication mechanics.

Applied Science Publishers, London, United Kingdom. 